\title{
A Note on a Formula of Riordan Involving Harmonic Numbers
}

\author{
J. A. Alonso-Carreón ${ }^{1}$, J. López-Bonilla ${ }^{1 \mathrm{a}}$, Gyan Bahadur Thapa ${ }^{2}$ \\ ${ }^{1}$ ESIME-Zacatenco, Instituto Politécnico Nacional, Edif. 5, 1er. Piso, Col. \\ Lindavista CP 07738, CDMX, México \\ ${ }^{2}$ Department of Applied Sciences, Pulcowk Campus, Institute of Engineering \\ Tribhuvan University, Nepal \\ acCorresponding author:jlopezb@ipn.mx
}

Received: Oct 10, 2018

Revised: $\operatorname{Dec} 28,2018$

Accepted: Jan 2, 2019

Abstract: We employ Stirling numbers of the second kind to prove a relation of Riordan involving harmonic numbers.

Keywords: Stirling numbers, Geometric series, Riordan's identity, Harmonic numbers.

\section{Introduction}

We know the Riordan's relation [7]:

$$
\sum_{k=1}^{n} \frac{x^{k}}{k}-H_{n}=\sum_{k=1}^{n}\left(\begin{array}{l}
n \\
k
\end{array}\right) \frac{(x-1)^{k}}{k}, \quad \forall x \in \mathbb{C},
$$

for the harmonic numbers [6]:

$$
H_{n}=\sum_{k=1}^{n} \frac{1}{k}
$$

It is usual to show (1) employing the geometric series and the binomial theorem of Newton; we observe that Agoh [1] also obtained this identity of Riordan. In Section 2, we exhibit an alternative proof of (1) via Stirling numbers $[6,7]$.

\section{Riordan's formula}

The generating function for the Stirling numbers of the second kind is given by [6]:

$$
\sum_{r=k}^{\infty} \frac{z^{r}}{r !} S_{r}^{[k]}=\frac{1}{k !}\left(e^{z}-1\right)^{k},
$$

with the property $[3,2]$ : 


$$
\sum_{k=1}^{n} k^{r-1}=\sum_{k=1}^{n}\left(\begin{array}{l}
n \\
k
\end{array}\right)(k-1) ! S_{r}^{[k]}
$$

then:

$$
\sum_{k=1}^{n} \frac{e^{k z}}{k}=\sum_{k=1}^{n} \frac{1}{k}\left(1+\sum_{r=1}^{\infty} \frac{k^{r} z^{r}}{r !}\right) \stackrel{(2)}{=} H_{n}+\sum_{r=1}^{\infty} \frac{z^{r}}{r !} \sum_{k=1}^{n} k^{r-1}
$$

that is:

$$
\sum_{k=1}^{n} \frac{e^{k z}}{k}-H_{n} \stackrel{(4)}{=} \sum_{k=1}^{n}\left(\begin{array}{l}
n \\
k
\end{array}\right)(k-1) ! \sum_{r=k}^{\infty} \frac{z^{r}}{r !} S_{r}^{[k]} \stackrel{(3)}{=} \sum_{k=1}^{n}\left(\begin{array}{l}
n \\
k
\end{array}\right) \frac{\left(e^{z}-1\right)^{k}}{k}
$$

where we can use $x=e^{z}$ to deduce (1), q. e. d.

Our procedure is simple and shows the connection between the harmonic numbers and the Stirling numbers of the second kind [5]. For $x=0$, the relation (1) implies the known expression of Euler [6]:

$$
H_{n}=\sum_{k=1}^{n}\left(\begin{array}{l}
n \\
k
\end{array}\right) \frac{(-1)^{k+1}}{k}
$$

Besides, from (1) is immediate the identity:

$$
\sum_{n=1}^{m} H_{n}=(m+1) \sum_{k=1}^{m} \frac{x^{k}}{k}-\sum_{k=1}^{m} x^{k}-\sum_{k=1}^{m}\left(\begin{array}{c}
m+1 \\
k+1
\end{array}\right) \frac{(x-1)^{k}}{k},
$$

where was applied the property $\sum_{n=k}^{m}\left(\begin{array}{l}n \\ k\end{array}\right)=\left(\begin{array}{c}m+1 \\ k+1\end{array}\right)$ [4]. The formula (6) with $x=0$ and $x=1$ implies [6]:

$$
\sum_{n=1}^{m} H_{n}=(m+1) H_{m}-m=(m+1)\left(H_{m+1}-1\right)=\sum_{k=1}^{m}\left(\begin{array}{c}
m+1 \\
k+1
\end{array}\right) \frac{(-1)^{k+1}}{k}
$$

Similarly from (1), it is simple to obtain for $x=1$ :

$$
\sum_{n=1}^{m} n H_{n}=\frac{m}{2}\left[(m+1) H_{m}+\frac{1}{2}(1-m)\right]=\frac{m(m+1)}{2}\left(H_{m+1}-\frac{1}{2}\right)
$$

\section{Conclusion}

Our procedure to prove (1) shows the important relationship between the harmonic numbers and the Stirling numbers of the second kind, without the participation of geometric series and the Newton's binomial theorem. 


\section{References}

[1] Agoh T (2016), On Miki's identity for Bernoulli numbers, Integers 16(73): 1-12.

[2] Boyadzhiev KN (2008/09), Power sum identities with generalized Stirling numbers, The Fibonacci Quart. 46-47, 4: 326-330.

[3] Butzer PL, Kilbas AA (2003), Stirling Functions of the Second Kind in the Setting of Difference and Fractional Calculus, Numerical Functional Analysis and Optimization, 24(7\&8): 673-711.

[4] Gould HW (1972), Combinatorial identities, Morgantown, W. Va.

[5] López-Bonilla J and López-Vázquez R (2017), Harmonic Numbers in terms of Stirling Numbers of the Second Kind, Prespacetime Journal, 8(2): 233-234.

[6] Quaintance J and Gould HW (2016), Combinatorial Identities for Stirling Numbers, World Scientific, Singapore.

[7] Riordan J (1968), Combinatorial identities, John Wiley \& Sons, New York. 